\title{
INFINITE DIMENSIONAL GEGENBAUER FUNCTIONALS
}

\author{
ABDESSATAR BARHOUMI \\ Department of Mathematics \\ Higher School of Sci. and Tech. of Hammam-Sousse \\ Sousse University, Sousse, Tunisia \\ E-mail: abdessatar.barhoumi@ipein.rnu.tn \\ HABIB OUERDIANE and ANIS RIAHI \\ Department of Mathematics \\ Faculty of Sciences of Tunis \\ University of Tunis El-Manar \\ 1060 Tunis, Tunisia \\ E-mail:habib.ouerdiane@fst.rnu.tn,a1riahi@yahoo.fr
}

\begin{abstract}
The paper is devoted to investigation of Gegenbauer white noise functionals. A particular attention is paid to the construction of the infinite dimensional Gegenbauer white noise measure $\mathcal{G}_{\beta}$, via the Bochner-Minlos theorem, on a suitable nuclear triple. Then we give the chaos decomposition of the $L^{2}$-space with respect to the measure $\mathcal{G}_{\beta}$ by using the so-called $\beta$-type Wick product.
\end{abstract}

1. Introduction. The white noise theory for Brownian motion was first introduced by $\mathrm{T}$. Hida, in the Gaussian case, in his celebrated lecture notes [6, 7]. Later, Kubo and Takenaka reformulated Hida's theory by taking different test function spaces and using the $S$-transform (see $[11,16]$ and references therein). For non-Gaussian white noise analysis, Y. Itô [8] constructed a Poissonian counterpart of Hida's theory and Kondratiev et al. [1, 10, 18] established a purely non-Gaussian distribution theory in infinite dimensional analysis by means of a normalized Laplace transform. (See also [5]). In [13, 9], a theory of the Lévy white noise analysis for the general case of Lévy processes is developed. See also the generalization and extension to various aspects, in $[17,14,15]$.

2000 Mathematics Subject Classification: Primary 60H40; Secondary 46A32, 46F25, 46G20.

Key words and phrases: Gegenbauer polynomials, Gegenbauer white noise measure, WienerItô isometry.

The paper is in final form and no version of it will be published elsewhere. 
In view of these different developments, it is natural to ask if a white noise theory can also be developed for other processes. In particular, since Gegenbauer processes are becoming increasingly important in many applications, it would be of interest to have a white noise theory for such processes. In fact, Gegenbauer processes are long memory processes and are characterized by an unbounded power spectral density at zero. From this last singularity property, one can observe that a natural tool to analyze such processes appears to be a generalization of the wavelet transform in quite different way from the Lévy processes. This was our motivation to intend to start a study on white noise functionals of general form on non-Lévy cases.

The development in our present paper is essentially the introduction of an infinite dimensional Gegenbauer white noise measure $\mathcal{G}_{\beta}, \beta>0$, and the chaos decomposition of an $L^{2}$-space with respect to the measure $\mathcal{G}_{\beta}$ by using an orthogonal system of infinite dimensional Gegenbauer polynomials. Such a decomposition is obtained by using the classical Gegenbauer polynomials as a counterpart of Hermite and Charlier polynomials in the Wiener-Itô chaos decomposition in Gaussian and Poisson white noise, respectively.

We shall use the following general formalism for classical polynomials. Let $\mu$ ba a probability measure on $\mathbf{R}$ with finite moments of all orders, such that the linear span of the monomials $x^{n}, n \geq 0$ is dense in $L^{2}(\mu)$. It is well known that there exists a complete system $\left\{P_{n}\right\}_{n=0}^{\infty}$ of orthogonal polynomials such that $P_{n}$ is a polynomial of degree $n$ with leading coefficient 1 and the following recursion formula is satisfied :

$$
\left(x-\alpha_{n}\right) P_{n}(x)=P_{n+1}(x)+w_{n} P_{n-1}(x) .
$$

Here, $\alpha_{n} \in \mathbf{R}$ and $w_{n} \geq 0$ for $n \geq 0$, and by convention $w_{0}=1, P_{-1}(x)=0$. The numbers $\alpha_{n}$ and $w_{n}$ are called Szegö-Jacobi parameters of $\mu$. (see [2, 3, 4, 19] for detailed development). The classical polynomials of Legendre, Chebyshev of the first kind, Chebyshev of the second kind and Gegenbauer are distinguished from their generating function, which involves the Fourier transform of their orthogonality measure. In this paper we focus on the $\beta$-type distribution and Gegenbauer polynomials.

The outline of our paper is the following. In Section 2, we review some fact about $\beta$-type distribution and Gegenbauer polynomials. Then we use the Gegenbauer functions to construct a standard nuclear triplet

$$
E_{\beta} \subset H:=L^{2}(]-1,1[, d x) \subset E_{\beta}^{\prime} .
$$

By the Bochner-Minlos theorem, we define the Gegenbauer white noise measure $\mathcal{G}_{\beta}$ on $\left(E_{\beta}^{\prime}, \mathcal{B}\left(E_{\beta}^{\prime}\right)\right)$ and we give some of its features. In section 3 , we define the $\beta$-type Wick product by means of an orthogonal system of infinite dimensional Gegenbauer polynomials and we investigate the chaos decomposition of the space $L^{2}\left(E_{\beta}^{\prime}, \mathcal{B}\left(E_{\beta}^{\prime}\right), \mathcal{G}_{\beta}\right)$.

\section{Gegenbauer white noise space}

2.1. Gegenbauer space in one dimension. Let $\mu_{\beta, \sigma}$ be the beta-type distribution with parameters $\beta>-\frac{1}{2}$ and $\sigma \in \mathbf{R}$ given by 


$$
\left\{\begin{array}{l}
d \mu_{\beta, \sigma}(x)=\frac{1}{|\sigma| \sqrt{\pi}} \frac{\Gamma(\beta+1)}{\Gamma\left(\beta+\frac{1}{2}\right)}\left(1-\frac{x^{2}}{\sigma^{2}}\right)^{\beta-\frac{1}{2}} \chi_{]-|\sigma|,|\sigma|[} d x, \\
d \mu_{\beta, 0}(x)=\delta_{0}(x),
\end{array}\right.
$$

where $\delta_{0}$ is the Dirac measure concentrated at the point 0 and $\Gamma(\cdot)$ is the Gamma function. For $\sigma=1$, we have the standard beta-type distribution with parameter $\beta$ :

$$
d \mu_{\beta}(x):=d \mu_{\beta, 1}(x)=\frac{1}{\sqrt{\pi}} \frac{\Gamma(\beta+1)}{\Gamma\left(\beta+\frac{1}{2}\right)}\left(1-x^{2}\right)^{\beta-\frac{1}{2}} \chi_{]-1,1[} d x .
$$

The following three important distributions are particular cases of (2.2).

- $d \mu_{\frac{1}{2}}(x)=\frac{1}{2} d x, \quad|x|<1$ : Uniform distribution;

- $d \mu_{0}(x)=\frac{1}{\pi} \frac{1}{\sqrt{1-x^{2}}} d x, \quad|x|<1$ : Arcsine distribution;

- $d \mu_{1}(x)=\frac{2}{\pi} \sqrt{1-x^{2}} d x, \quad|x|<1$ : Semi-circle distribution.

From the paper [3], we recall the following useful background. Apply the GramSchmidt orthogonalization process to the sequence $\left\{1, x, x^{2}, \cdots, x^{n}, \cdots\right\}$ to get a sequence $\left\{P_{\beta, n} ; n=0,1,2, \cdots\right\}$ of orthogonal polynomials in $L^{2}\left(\mu_{\beta}\right)$. Here $P_{\beta, 0}(x)=1$ and for $\beta>0, P_{\beta, n}$ is the Gegenbauer polynomial given by

$$
P_{\beta, n}(x)=\frac{n !}{2^{n}(\beta)_{n}} \sum_{k=0}^{[n / 2]}(-1)^{n} 2^{n-2 k}\left(\begin{array}{c}
-\beta \\
n-k
\end{array}\right)\left(\begin{array}{c}
n-k \\
k
\end{array}\right) x^{n-2 k},
$$

where the shifted factorials are given by

$$
(a)_{0}=1 ;(a)_{k}=a(a+1) \cdots(a+k-1)=\frac{\Gamma(a+k)}{\Gamma(a)}, \quad \forall k \in \mathbf{N},
$$

and for $\delta \in \mathbf{R}, p \in \mathbf{N}^{*}$, we have

$$
\left(\begin{array}{l}
\delta \\
p
\end{array}\right)=\frac{\delta(\delta-1) \ldots(\delta-p+1)}{p !} .
$$

For the Szegö-Jacobi parameters, since the measure $\mu_{\beta}$ is symmetric we have

$$
\alpha_{n}=0 \quad \text { and } \quad w_{n}=\frac{n(n-1+2 \beta)}{4(n+\beta)(n-1+\beta)}, \quad n \geq 0 .
$$

It is well-known that, according to (1.1), these polynomials $P_{\beta, n}$ satisfy the recursion formula

$$
x P_{\beta, n}(x)=P_{\beta, n+1}(x)+w_{n} P_{\beta, n-1}(x) .
$$

We recall that the generating function $\psi_{\mu_{\beta}}$ associated to the measure $\mu_{\beta}$ is given by

$$
\psi_{\mu_{\beta}}(t, x)=\frac{1}{\left(1-2 x t+t^{2}\right)^{\beta}}=\sum_{n=0}^{\infty} \frac{2^{n}(\beta)_{n}}{n !} t^{n} P_{\beta, n}(x) .
$$

REMARK 2.1. Note that the Legendre polynomials and Chebyshev polynomials of the second kind are the special cases of Gegenbauer's, with $\beta=\frac{1}{2}$ and $\beta=1$ respectively. 
For the case $\beta=0$, we recall the Chebyshev polynomials of the first $\operatorname{kind} T_{n}$ given by

$$
T_{n}(x)=x^{n}+\frac{1}{2^{n}} \sum_{k=0}^{[n / 2]}(-1)^{k} \frac{n}{k} 2^{n-2 k}\left(\begin{array}{c}
n-k-1 \\
k-1
\end{array}\right) x^{n-2 k} .
$$

Furthermore, the generating function of the arcsine distribution is given by

$$
\psi_{\mu_{0}}(t, x)=\frac{1-t^{2}}{1-2 x t+t^{2}}=\sum_{n=0}^{+\infty} 2^{n} T_{n}(x) t^{n} .
$$

A detailed development of this special case will appear in a future paper. Thus we assume that $\beta>0$ from now on.

The Bessel function of the first kind of order $\alpha>-\frac{1}{2}$ can be defined by (see [19])

$$
J_{\alpha}(x)=\left(\frac{x}{2}\right)^{\alpha} \sum_{k=0}^{+\infty} \frac{(-1)^{k}}{k ! \Gamma(\alpha+k+1)}\left(\frac{x}{2}\right)^{2 k}, \quad x>0 .
$$

Moreover, we have the following Poisson-Mehler integral representation

$$
J_{\alpha}(x)=\frac{1}{\sqrt{\pi} \Gamma\left(\alpha+\frac{1}{2}\right)}\left(\frac{x}{2}\right)^{\alpha} \int_{-1}^{1}\left(1-t^{2}\right)^{\alpha-\frac{1}{2}} e^{i x t} d t .
$$

The normalized Bessel function of order $\alpha>-\frac{1}{2}$ is given by

$$
j_{\alpha}(x)=\left\{\begin{array}{ccc}
2^{\alpha} \Gamma(\alpha+1) \frac{J_{\alpha}(x)}{x^{\alpha}} & \text { if } & x \neq 0 \\
1 & \text { if } & x=0
\end{array}\right.
$$

Using (2.3) and (2.4), the Fourier transform of the beta-type distribution, in Eq. (2.1) is given by

$$
\widehat{\mu}_{\beta, \sigma}(x)=\int_{\mathbf{R}} e^{i x t} d \mu_{\beta, \sigma}(t)=j_{\beta}(|\sigma| x), \quad x \in \mathbf{R} .
$$

2.2. Construction of the Gegenbauer white noise space. For simplicity, put $I=]-1,1[$. From the Favard theorem [4], one can easily obtain

$$
\left\|P_{\beta, n}\right\|_{L^{2}\left(I, \mu_{\beta}\right)}^{2}=\frac{n !}{4^{n}} \frac{(2 \beta)_{n}}{(\beta)_{n}(\beta+1)_{n}}=: M_{\beta, n},
$$

therefore, we define the corresponding Gegenbauer functions $\mathcal{H}_{\beta, n}(x)$ by

$$
\mathcal{H}_{\beta, n}(x)=\left(\frac{\Gamma(\beta+1)}{\sqrt{\pi} \Gamma\left(\beta+\frac{1}{2}\right) M_{\beta, n}}\right)^{\frac{1}{2}} P_{\beta, n}(x)\left(1-x^{2}\right)^{\frac{2 \beta-1}{4}} .
$$

This gives an orthonormal basis $\left\{\mathcal{H}_{\beta, n}, n=0,1,2, \cdots\right\}$ for $H:=L^{2}(I, d x)$.

Lemma 2.2. Define the operator $A_{\beta}$, on $H$, by

$$
A_{\beta}=\left(x^{2}-1\right) \frac{d^{2}}{d x^{2}}+2 x \frac{d}{d x}-\left(\beta-\frac{1}{2}\right)^{2} \frac{x^{2}}{x^{2}-1}+2 .
$$

Then the Gegenbauer functions $\mathcal{H}_{\beta, n}$ are eigenvectors of $A_{\beta}$, namely,

$$
A_{\beta} \mathcal{H}_{\beta, n}=\lambda_{\beta, n} \mathcal{H}_{\beta, n}
$$


with

$$
\lambda_{\beta, n}=\beta+\frac{3}{2}+n(n+2 \beta), \quad n=0,1,2, \cdots
$$

Moreover, for any $p>\frac{1}{4}, A_{\beta}^{-p}$ is a Hilbert-Schmidt operator satisfying

$$
\left\|A_{\beta}^{-p}\right\|_{H S}^{2}=\sum_{n=0}^{\infty} \lambda_{\beta, n}^{-2 p}<\infty .
$$

Proof. For simplicity, we put

$$
f_{\beta, n}(x)=\left(1-x^{2}\right)^{\frac{2 \beta-1}{4}} P_{\beta, n}(x) .
$$

Then, it is easy to see that

$$
\begin{aligned}
f_{\beta, n}^{\prime \prime}(x)-\frac{2 x}{1-x^{2}} & f_{\beta, n}^{\prime}(x)-\left(\beta-\frac{1}{2}\right)^{2} \frac{x^{2}}{\left(1-x^{2}\right)^{2}} f_{\beta, n}(x)-\frac{2}{1-x^{2}} f_{\beta, n}(x) \\
& =\left(1-x^{2}\right)^{\frac{\beta}{2}-\frac{1}{4}}\left(P_{\beta, n}^{\prime \prime}(x)-\frac{(2 \beta+1) x}{1-x^{2}} P_{\beta, n}^{\prime}(x)-\frac{\beta+\frac{3}{2}}{1-x^{2}} P_{\beta, n}(x)\right) .
\end{aligned}
$$

On the other hand, $P_{\beta, n}$ satisfies the differential equation

$$
P_{\beta, n}^{\prime \prime}(x)-\frac{(2 \beta+1) x}{1-x^{2}} P_{\beta, n}^{\prime}(x)+\frac{n(n+2 \beta)}{1-x^{2}} P_{\beta, n}(x)=0 .
$$

Hence, we have

$$
\left(x^{2}-1\right) f_{\beta, n}^{\prime \prime}(x)+2 x f_{n}^{\prime}(x)-\left(\beta-\frac{1}{2}\right)^{2} \frac{x^{2}}{1-x^{2}} f_{\beta, n}(x)+2 f_{\beta, n}(x)=\lambda_{\beta, n} f_{\beta, n}(x),
$$

which gives (2.6). The second statement is straightforward.

Now, for each $p \in \mathbf{R}$, define a norm $|\cdot|_{p}$ on $H$ by

$$
|f|_{p}=\left|A_{\beta}^{p}\right| f \|_{0}=\left(\sum_{n=0}^{\infty} \lambda_{\beta, n}^{2 p}\left\langle|f|, \mathcal{H}_{\beta, n}\right\rangle^{2}\right)^{1 / 2}, \quad f \in H,
$$

where $|\cdot|_{0}$ and $\langle\cdot, \cdot\rangle$ are, respectively, the norm and the inner product of $H$. For $p \geq 0$, let $E_{p, \beta}$ be the Hilbert space consisting of all $f \in H$ with $|f|_{p}<\infty$, and $E_{-p, \beta}$ the completion of $H$ with respect to $|\cdot|_{-p}$. Since $A_{\beta}^{-1}$ is of Hilbert-Schmidt type, identifying $H$ with its dual space we come to the real standard nuclear triple

$$
E_{\beta}:=\bigcap_{p \geq 0} E_{p, \beta} \subset H \subset \bigcup_{p \geq 0} E_{-p, \beta}:=E_{\beta}^{\prime} .
$$

Being compatible to the inner product of $H$, the canonical bilinear form on $E_{\beta}^{\prime} \times E_{\beta}$ is denoted by $\langle\cdot, \cdot\rangle$ again.

Lemma 2.3. The function

$$
C_{\beta}(\varphi)=j_{\beta}(\langle\varphi\rangle), \varphi \in E_{\beta},
$$

is a characteristic function on $E_{\beta}$, where $\langle\varphi\rangle=\int_{I} \varphi(x) d x$. 
Proof. Obviously $C_{\beta}$ is continuous on $E_{\beta}$ and $C_{\beta}(0)=1$. We shall prove that $C_{\beta}$ is positive definite. Given $\alpha_{1}, \alpha_{2}, \cdots, \alpha_{n} \in \mathbf{C}$ and $\varphi_{1}, \varphi_{2}, \cdots, \varphi_{n} \in E_{\beta}$, we have

$$
\begin{aligned}
\sum_{j, k=1}^{n} \alpha_{j} \bar{\alpha}_{k} C_{\beta}\left(\varphi_{j}-\varphi_{k}\right) & =\sum_{j, k=1}^{n} \alpha_{j} \bar{\alpha}_{k} \frac{\Gamma(\beta+1)}{\sqrt{\pi} \Gamma\left(\beta+\frac{1}{2}\right)} \int_{-1}^{1}\left(1-t^{2}\right)^{\beta-\frac{1}{2}} e^{i t\left\langle\varphi_{j}-\varphi_{k}\right\rangle} d t \\
& =\frac{\Gamma(\beta+1)}{\sqrt{\pi} \Gamma\left(\beta+\frac{1}{2}\right)} \int_{-1}^{1}\left(1-t^{2}\right)^{\beta-\frac{1}{2}}\left|A_{t}\right|^{2} d t \geq 0,
\end{aligned}
$$

where $A_{t}=\sum_{j=1}^{n} \alpha_{j} \exp \left(i t\left\langle\varphi_{j}\right\rangle\right)$.

An application of the Bochner-Minlos theorem leads us to the following.

Definition 2.4. The probability measure $\mathcal{G}_{\beta}$, on $E_{\beta}^{\prime}$, whose characteristic function is $C_{\beta}$ given by (2.8), is called the Gegenbauer white noise measure with parameter $\beta$. The probability space $\left(E_{\beta}^{\prime}, \mathcal{G}_{\beta}\right)$ is called the Gegenbauer white noise space.

Proposition 2.5. For $\xi \in E_{\beta}$, let $X_{\xi}$ be the random variable defined, on $\left(E_{\beta}^{\prime}, \mathcal{B}\left(E_{\beta}^{\prime}\right), \mathcal{G}_{\beta}\right)$, by

$$
X_{\xi}(w):=\langle w, \xi\rangle,
$$

where $\mathcal{B}\left(E_{\beta}^{\prime}\right)$ is the cylinder $\sigma$-algebra on $E_{\beta}^{\prime}$. If $\langle\xi\rangle \geq 0$, then $X_{\xi}$ has a beta-type distribution with parameters $\beta$ and $\langle\xi\rangle$.

Proof. By using (2.1), (2.5) and (2.8), we have

$$
\int_{E_{\beta}^{\prime}} e^{i \lambda X_{\xi}(w)} d \mathcal{G}_{\beta}(w)=j_{\beta}(\langle\lambda \xi\rangle)=\widehat{\mu}_{\beta,\langle\xi\rangle}(\lambda), \quad \lambda \in \mathbf{R} .
$$

This proves that the distribution of the random variable $X_{\xi}$ is the probability measure $\mu_{\beta,\langle\xi\rangle}$ on $\mathbf{R}$.

REMARK 2.6. Indeed, for $t \in \mathbf{R}^{*}$, setting in (2.8) $\varphi=\lambda \chi_{[0 \wedge t, 0 \vee t]}$, where $0 \wedge t=\min (0, t)$ and $0 \vee t=\max (0, t)$, we obtain

$$
C_{\beta}(\varphi)= \begin{cases}j_{\beta}(\lambda|t|) & \text { if }|t|<1 \\ j_{\beta}(\lambda) & \text { if }|t| \geq 1\end{cases}
$$

This function coincides with the Fourier transform of the measure $\mu_{\beta,|t|}$ if $|t|<1$ and the measure $\mu_{\beta}$ if $|t| \geq 1$. On the probability space $\left(E_{\beta}^{\prime}, \mathcal{B}\left(E_{\beta}^{\prime}\right), \mathcal{G}_{\beta}\right)$, the random variable $X_{t}:=\left\langle\cdot, \chi_{[0 \wedge t, 0 \vee t]}\right\rangle$ has a beta-type distribution. So the family of random variables

$$
X=\left\{X_{t}, t \in \mathbf{R}\right\}, \quad X_{0}=0
$$

is called a Gegenbauer process. The image of the Gegenbauer white noise measure $\mathcal{G}_{\beta}$ under the random variable $X_{t}(\cdot)$ is the beta-type distribution $\mu_{\beta}$ if $|t| \geq 1$ and the beta-type distribution $\mu_{\beta,|t|}$ if $|t|<1$. Then, for $t>s>0$, the characteristic function $\mathbf{E}\left[\exp \left\{i \lambda\left(X_{t}-X_{s}\right)\right\}\right]$ of $X_{t}-X_{s}$ has the following form:

$$
\mathbf{E}\left[\exp \left\{i \lambda\left(X_{t}-X_{s}\right)\right\}\right]= \begin{cases}j_{\beta}(\lambda(t-s)) & \text { if } s, t \in(0,1), \\ j_{\beta}(\lambda(1-s)) & \text { if } 0<s<1 \leq t, \\ j_{\beta}(0)=1 & \text { if } s, t>1\end{cases}
$$


We conclude that for $s, t \in(0,1)$ the random variable $X_{t}-X_{s}$ has the beta-type distribution with parameters $\beta$ and $t-s$. This gives an essence of our approach for defining the Gegenbauer white noise functionals.

3. Chaos decomposition of the white noise gegenbauer space. Let $L^{2}\left(E_{\beta}^{\prime}, \mathcal{G}_{\beta}\right)$ be the real Hilbert space of square $\mathcal{G}_{\beta}$-integrable functions with norm denoted by $\|\cdot\|_{0}$. In the remainder of this paper we shall discuss some properties of the white noise Gegenbauer space $L^{2}\left(E_{\beta}^{\prime}, \mathcal{G}_{\beta}\right)$.

LEMma 3.1. The measure $\mathcal{G}_{\beta}$ satisfies the moment condition:

$$
\int_{E_{\beta}^{\prime}}\langle w, \xi\rangle^{n} d \mathcal{G}_{\beta}(w)<\infty
$$

for any $\xi \in E_{\beta}$ satisfying $\langle\xi\rangle \geq 0$ and $n \in N$. More precisely, we have:

1.

$$
\int_{E_{\beta}^{\prime}}\langle w, \xi\rangle^{2 n} d \mathcal{G}_{\beta}(w)=\frac{\left(\frac{1}{2}\right)_{n}}{(\beta+1)_{n}}\langle\xi\rangle^{2 n}
$$

2. $\int_{E_{\beta}^{\prime}}\langle w, \xi\rangle^{2 n+1} d \mathcal{G}_{\beta}(w)=0$.

Proof. By using proposition 2.5, we get

$$
\int_{E_{\beta}^{\prime}}\langle w, \xi\rangle^{2 n} d \mathcal{G}_{\beta}(w)=\int_{\mathbf{R}} t^{2 n} d \mu_{\beta,\langle\xi\rangle}(t)=\frac{\left(\frac{1}{2}\right)_{n}}{(\beta+1)_{n}}\langle\xi\rangle^{2 n}
$$

This proves the first statement. The second statement is obvious from the symmetry of the measure $\mu_{\beta,\langle\xi\rangle}$.

From the above Lemma, the linear functional $w \mapsto X_{\xi}(w), \xi \in E_{\beta}$, belongs to $L^{2}\left(E_{\beta}^{\prime}, \mathcal{G}_{\beta}\right)$. Now, for each $\phi \in H=L^{2}(I, d t)$, choose a sequence $\left\{\phi_{n}\right\} \subset E_{\beta}$ so that $\phi_{n} \rightarrow \phi$ in $H$. Then it follows from (3.1) that $\left\{\left\langle\cdot, \phi_{n}\right\rangle\right\}$ forms a Cauchy sequence in $L^{2}\left(E_{\beta}^{\prime}, \mathcal{G}_{\beta}\right)$. Denote by $\langle\cdot, \phi\rangle$ the $L^{2}$-limit of $\left\{\left\langle\cdot, \phi_{n}\right\rangle\right\}$. When $\phi=\chi_{(s, t)}$, the indicator of $(s, t)$, the characteristic function of $\langle\cdot, \phi\rangle$ is exactly the same as the one in (2.9). So the Gegenbauer process $X$ on $\left(E_{\beta}^{\prime}, \mathcal{B}\left(E_{\beta}^{\prime}\right), \mathcal{G}_{\beta}\right)$ can be represented by

$$
X(t ; w)= \begin{cases}\left\langle w, \chi_{(0, t)}\right\rangle & \text { if } t \geq 0, \\ -\left\langle w, \chi_{(t, 0)}\right\rangle & \text { if } t<0, \quad w \in E_{\beta}^{\prime} .\end{cases}
$$

Taking the time derivative formally, we get

$$
\dot{X}(t ; w)=w(t), \quad w \in E_{\beta}^{\prime} .
$$

Thus the elements of $E_{\beta}^{\prime}$ can be regarded as the sample paths of the Gegenbauer white noise, and members of $L^{2}\left(E_{\beta}^{\prime}, \mathcal{G}_{\beta}\right)$ are called Gegenbauer white noise functionals.

Now, we introduce polynomial functions on the white noise Gegenbauer space $\left(E_{\beta}^{\prime}, \mathcal{G}_{\beta}\right)$. Let $\mathcal{P}_{n}\left(E_{\beta}^{\prime}\right)$ be the space of finite linear combinations of functions of the form

$$
w \mapsto\left\langle w, \xi_{1}\right\rangle \cdots\left\langle w, \xi_{n}\right\rangle=\left\langle w^{\otimes n}, \xi_{1} \otimes \cdots \otimes \xi_{n}\right\rangle, \quad w \in E_{\beta}^{\prime}, \quad \xi_{1}, \cdots, \xi_{n} \in E_{\beta} .
$$


An element of the algebraic sums

$$
\mathcal{P}\left(E_{\beta}^{\prime}\right)=\sum_{n=0}^{\infty} \mathcal{P}_{n}\left(E_{\beta}^{\prime}\right)
$$

is called a polynomial on the space $\left(E_{\beta}^{\prime}, \mathcal{G}_{\beta}\right)$.

From Eq. (2.7) one can see that the nuclear space $E_{\beta}$ is closed under the absolute value. This enables us to introduce a Wick product in the following way.

Definition 3.2. For $w \in E_{\beta}^{\prime}$ and $n=0,1,2, \cdots$, we define the $\beta$-type Wick product $: w^{\otimes n}:_{\beta} \in E_{\beta}^{\prime \widehat{\otimes} n}$ as the linear functional on $E_{\beta}^{\widehat{\otimes} n}$ characterized by

$$
\left\langle: w^{\otimes n}:_{\beta}, \varphi^{\otimes n}\right\rangle=|\varphi|_{0}^{n} P_{\beta, n}\left(\frac{\langle w,|\varphi|\rangle}{\langle|\varphi|\rangle}\right), \quad \varphi \in E_{\beta},
$$

and for any orthogonal vectors $\xi_{1}, \cdots, \xi_{k} \in E_{\beta}$ and nonnegative integers $n_{j}$ 's such that $n_{1}+\cdots+n_{k}=n$, we have

$$
\left\langle: w^{\otimes n}:_{\beta}, \xi_{1}^{\otimes n_{1}} \widehat{\otimes} \cdots \widehat{\otimes} \xi_{k}^{\otimes n_{k}}\right\rangle=\left\langle: w^{\otimes n_{1}}:_{\beta}, \xi_{1}^{\otimes n_{1}}\right\rangle \cdots\left\langle: w^{\otimes n_{k}}:_{\beta}, \xi_{k}^{\otimes n_{k}}\right\rangle .
$$

LEMMA 3.3. We have the following statements:

1.

$$
\int_{E_{\beta}^{\prime}}\left\langle: w^{\otimes n}:_{\beta}, \xi^{\otimes n}\right\rangle\left\langle: w^{\otimes m}:_{\beta}, \xi^{\otimes m}\right\rangle d \mathcal{G}_{\beta}(w)=M_{\beta, n}|\xi|_{0}^{2 n} \delta_{m, n}, \quad \xi \in E_{\beta}
$$

2. $\int_{E_{\beta}^{\prime}}\left\langle: w^{\otimes m}:_{\beta}, \xi^{\otimes m}\right\rangle\left\langle: w^{\otimes n}:{ }_{\beta}, \eta^{\otimes n}\right\rangle d \mathcal{G}_{\beta}(w)=M_{\beta, n}\langle\xi, \eta\rangle^{n} \delta_{m, n} ; \quad \xi, \eta \in E_{\beta}$.

3. For all $\phi_{n}, \psi_{n} \in E_{\beta}^{\widehat{\otimes} n}$, we have :

$$
\int_{E_{\beta}^{\prime}}\left\langle: w^{\otimes n}:_{\beta}, \phi_{n}\right\rangle\left\langle: w^{\otimes m}:_{\beta}, \psi_{m}\right\rangle d \mathcal{G}_{\beta}(w)=M_{\beta, n}\left\langle\phi_{n}, \psi_{n}\right\rangle \delta_{m, n} .
$$

Proof. 1. For $\xi \in E_{\beta}, \xi \neq 0$, the image of the Gegenbauer white noise measure $\mathcal{G}_{\beta}$ under the map

$$
w \mapsto\left\langle w, \frac{|\xi|}{\langle|\xi|\rangle}\right\rangle \in \mathbf{R}, \quad w \in E_{\beta}^{\prime},
$$

is the beta-type distribution $\mu_{\beta}$. Then we have

$$
\begin{aligned}
\int_{E_{\beta}^{\prime}}\left\langle: w^{\otimes n}:_{\beta}, \xi^{\otimes n}\right\rangle\left\langle: w^{\otimes m}:_{\beta}, \xi^{\otimes m}\right\rangle d \mathcal{G}_{\beta}(w) \\
=|\xi|_{0}^{n+m} \int_{I} P_{\beta, n}(t) P_{\beta, m}(t) d \mu_{\beta}(t)=M_{\beta, n}|\xi|_{0}^{2 n} \delta_{m, n} .
\end{aligned}
$$

2. It is sufficient to prove the identity under the assumption $|\xi|_{0}=|\eta|_{0}=1$. Taking a unit vector $\zeta \in E_{\beta}$ such that $\langle\xi, \zeta\rangle=0$, we may write

$$
\eta=\alpha \xi+\lambda \zeta, \quad \alpha^{2}+\lambda^{2}=1
$$

and we have

$$
\left\langle: w^{\otimes n}:_{\beta}, \eta^{\otimes n}\right\rangle=\sum_{k=0}^{n}\left(\begin{array}{l}
n \\
k
\end{array}\right) \alpha^{n-k} \lambda^{k}\left\langle: w^{\otimes(n-k)}:_{\beta}, \xi^{\otimes(n-k)}\right\rangle\left\langle: w^{\otimes k}:_{\beta}, \zeta^{\otimes k}\right\rangle .
$$


Then we get

$$
\begin{aligned}
\int_{E_{\beta}^{\prime}}\left\langle: w^{\otimes m}:_{\beta}, \xi^{\otimes m}\right\rangle\left\langle: w^{\otimes n}:_{\beta}, \eta^{\otimes n}\right\rangle d \mathcal{G}_{\beta}(w)=\sum_{k=0}^{n}\left(\begin{array}{c}
n \\
k
\end{array}\right) \alpha^{n-k} \lambda^{k} \\
\times \int_{E_{\beta}^{\prime}}\left\langle: w^{\otimes m}:_{\beta}, \xi^{\otimes m}\right\rangle\left\langle: w^{\otimes(n-k)}:_{\beta}, \xi^{\otimes(n-k)}\right\rangle\left\langle: w^{\otimes k}:_{\beta}, \zeta^{\otimes k}\right\rangle d \mathcal{G}_{\beta}(w) .
\end{aligned}
$$

On the other hand, by using the independence of the two random variables $\langle\cdot, \xi\rangle$ and $\langle\cdot, \zeta\rangle$, we obtain

$$
\begin{aligned}
& \int_{E_{\beta}^{\prime}}\left\langle: w^{\otimes m}:_{\beta}, \xi^{\otimes m}\right\rangle\left\langle: w^{\otimes(n-k)}:_{\beta}, \xi^{\otimes(n-k)}\right\rangle\left\langle: w^{\otimes k}:_{\beta}, \zeta^{\otimes k}\right\rangle d \mathcal{G}_{\beta}(w) \\
= & \int_{E_{\beta}^{\prime}}\left\langle: w^{\otimes m}:_{\beta}, \xi^{\otimes m}\right\rangle\left\langle: w^{\otimes(n-k)}:_{\beta}, \xi^{\otimes(n-k)}\right\rangle d \mathcal{G}_{\beta}(w) \int_{E_{\beta}^{\prime}}\left\langle: w^{\otimes k}:_{\beta}, \zeta^{\otimes k}\right\rangle d \mathcal{G}_{\beta}(w) .
\end{aligned}
$$

Therefore the last integral is equal to 0 unless $k=0$ and is equal to 1 if $k=0$. Hence,

$$
\begin{aligned}
& \int_{E_{\beta}^{\prime}}\left\langle: w^{\otimes m}:_{\beta}, \xi^{\otimes m}\right\rangle\left\langle: w^{\otimes n}:_{\beta}, \eta^{\otimes n}\right\rangle d \mathcal{G}_{\beta}(w) \\
&=\alpha^{n} \int_{E_{\beta}^{\prime}}\left\langle: w^{\otimes m}: \beta, \xi^{\otimes m}\right\rangle\left\langle: w^{\otimes n}:_{\beta}, \xi^{\otimes n}\right\rangle d \mathcal{G}_{\beta}(w) .
\end{aligned}
$$

Applying (3.4), we conclude that

$$
\int_{E_{\beta}^{\prime}}\left\langle: w^{\otimes m}:_{\beta}, \xi^{\otimes m}\right\rangle\left\langle: w^{\otimes n}:_{\beta}, \eta^{\otimes n}\right\rangle d \mathcal{G}_{\beta}(w)=M_{\beta, n} \alpha^{n} \delta_{m, n} .
$$

Since $\alpha=\langle\xi, \eta\rangle$, we have completed the proof of 2 .

3. The statement follows from the second assertion by considering $\phi_{n}$ and $\psi_{n}$ as linear combinations of elements of the form $\xi^{\otimes n}, \xi \in E_{\beta}$.

Proposition 3.4. For two polynomials $\phi, \psi \in \mathcal{P}\left(E_{\beta}^{\prime}\right)$, given respectively by

$$
\phi(w)=\sum_{n=0}^{\infty}\left\langle: w^{\otimes n}:_{\beta}, \phi_{n}\right\rangle, \quad \psi(w)=\sum_{n=0}^{\infty}\left\langle: w^{\otimes n}:_{\beta}, \psi_{n}\right\rangle,
$$

we have

$$
\int_{E_{\beta}^{\prime}} \phi(w) \psi(w) d \mathcal{G}_{\beta}(w)=\sum_{n=0}^{\infty} M_{\beta, n}\left\langle\phi_{n}, \psi_{n}\right\rangle .
$$

In particular, the $L^{2}$-norm of $\phi$, with respect to $\mathcal{G}_{\beta}$, is given by

$$
\|\phi\|_{0}^{2}=\sum_{n=0}^{\infty} M_{\beta, n}\left|\phi_{n}\right|_{0}^{2} .
$$

Proof. The statement follows from Lemma 3.3.

The $\beta$-Fock space $\mathcal{F}_{\beta}(H)$ over $H$ is defined as the weighted direct sum of the $n$-th symmetric tensor powers $H^{\widehat{\otimes} n}, n \in \mathbf{N}$,

$$
\mathcal{F}_{\beta}(H):=\bigoplus_{n=0}^{+\infty} M_{\beta, n} H^{\widehat{\otimes} n}
$$


Thus $\mathcal{F}_{\beta}(H)$ consists of sequences $\vec{f}=\left(f^{(0)}, f^{(1)}, \cdots\right)$ such that, for any $n \in \mathbf{N}, f^{(n)} \in$ $H^{\widehat{\otimes} n}$ and

$$
\|\vec{f}\|_{\mathcal{F}_{\beta}(H)}^{2}=\sum_{n=0}^{+\infty} M_{\beta, n}\left\|f^{(n)}\right\|_{H^{\hat{\otimes} n}}^{2}<\infty .
$$

Proposition 3.5. For $\mathbf{n}=\left(n_{0}, n_{1}, n_{2}, \cdots\right)$ with $|\mathbf{n}|=n_{0}+n_{1}+n_{2}+\cdots=n<\infty$ we put

$$
e_{\beta, \mathbf{n}}:=\left(0, \cdots, \frac{\mathcal{H}_{\beta, n_{0}}^{\otimes n_{0}} \widehat{\otimes} \mathcal{H}_{\beta, n_{1}}^{\otimes n_{1}} \widehat{\otimes} \cdots}{\sqrt{M_{\beta, n_{0}} M_{\beta, n_{1}} \cdots}}, 0, \cdots\right),
$$

where the non-zero element occurs in the $(n+1)$-th place. Then, $\left\{e_{\beta, \mathbf{n}},|\mathbf{n}|=n\right\}$ constitute a complete orthonormal basis for $\mathcal{F}_{\beta}(H)$. Moreover, if we put

$$
\phi_{\beta, \mathbf{n}}(w):=\left(M_{\beta, n_{0}} M_{\beta, n_{1}} \cdots\right)^{-1 / 2}\left\langle: w^{\otimes n}:_{\beta}, \mathcal{H}_{\beta, n_{0}}^{\otimes n_{0}} \widehat{\otimes} \mathcal{H}_{\beta, n_{1}}^{\otimes n_{1}} \widehat{\otimes} \cdots\right\rangle,
$$

then $\left\{\phi_{\beta, \mathbf{n}},|\mathbf{n}|<\infty\right\}$ forms a complete orthonormal basis for $L^{2}\left(E_{\beta}^{\prime}, \mathcal{G}_{\beta}\right)$.

Proof. The proof is obvious by the definition of the $\beta$-Fock space.

THEOREM 3.6. For each $F \in L^{2}\left(E_{\beta}^{\prime}, \mathcal{G}_{\beta}\right)$, there exists a unique sequence $\vec{f}=\left(f^{(n)}\right)_{n=0}^{\infty}$ $\in \mathcal{F}_{\beta}(H)$ such that

$$
F=\sum_{n=0}^{+\infty}\left\langle: \cdot{ }^{\otimes n}:_{\beta}, f^{(n)}\right\rangle
$$

in the $L^{2}-$ sense. Conversely, for any $\vec{f}=\left(f^{(n)}\right)_{n=0}^{\infty} \in \mathcal{F}_{\beta}(H)$, (3.5) defines a function on $L^{2}\left(E_{\beta}^{\prime}, \mathcal{G}_{\beta}\right)$. In that case,

$$
\|F\|_{L^{2}\left(E_{\beta}^{\prime}, \mathcal{G}_{\beta}\right)}^{2}=\sum_{n=0}^{+\infty} M_{\beta, n}\left\|f^{(n)}\right\|_{H^{\hat{\otimes} n}}^{2}=\|\vec{f}\|_{\mathcal{F}_{\beta}(H)}^{2} .
$$

The following unitary operator is called the Wiener-Itô isometry:

$$
I_{\beta}: \mathcal{F}_{\beta}(H) \rightarrow L^{2}\left(E_{\beta}^{\prime}, \mathcal{G}_{\beta}\right), \quad\left(f^{(n)}\right)_{n=0}^{\infty} \mapsto F
$$

Proof. It is easy to see that the set

$$
\mathcal{P}\left(E_{\beta}^{\prime}\right)=\left\{\phi, \phi(w)=\sum_{k=0}^{n}\left\langle: w^{\otimes k}:_{\beta}, \phi^{(k)}\right\rangle, \phi^{(k)} \in E_{\beta}^{\otimes k}, w \in E_{\beta}^{\prime}, n \in \mathbf{N}\right\}
$$

of smooth continuous polynomials on $E_{\beta}^{\prime}$ is continuously and densely embedded in $L^{2}\left(E_{\beta}^{\prime}, \mathcal{G}_{\beta}\right)$. Then, for any $F \in L^{2}\left(E_{\beta}^{\prime}, \mathcal{G}_{\beta}\right)$, there exists a unique sequence $\vec{f}=\left(f^{(n)}\right)_{n=0}^{\infty}$ $\in \mathcal{F}_{\beta}(H)$ such that

$$
F=\sum_{n=0}^{+\infty}\left\langle:{ }^{\otimes n}:_{\beta}, f^{(n)}\right\rangle
$$

It follows that

$$
\begin{aligned}
\|F\|_{L^{2}\left(E_{\beta}^{\prime}, \mathcal{G}_{\beta}\right)}^{2} & =\int_{E_{\beta}^{\prime}}\left(\sum_{n=0}^{+\infty}\left\langle: w^{\otimes n}:_{\beta}, f^{(n)}\right\rangle\right)^{2} d \mathcal{G}_{\beta}(w) \\
& =\sum_{n=0}^{+\infty} M_{\beta, n}\left\langle f^{(n)}, f^{(n)}\right\rangle_{H^{\hat{\otimes} n}}=\|\vec{f}\|_{\mathcal{F}_{\beta}(H)}^{2},
\end{aligned}
$$


where Proposition 3.4 is taken into account. The second part of the Theorem is straightforward.

Acknowledgments. We are grateful to the anonymous referee for the detailed comments and suggestions.

\section{References}

[1] S. Albeverio, Yu. L. Daletsky, Yu. G. Kondratiev and L. Streit, Non-Gaussian infinite dimensional analysis, J. Funct. Anal. 138 (1996), 311-350.

[2] N. Asai, I. Kubo and H.-H. Kuo, Multiplicative renormalization and generating functions I, Taiwanese Journal of Mathematics 7 (2003), 89-101.

[3] N. Asai, I. Kubo and H.-H. Kuo, Multiplicative renormalization and generating functions II, Taiwanese Journal of Mathematics 8 (2004), 583-628.

[4] T. S. Chihara, An Introduction to Orthogonal Polynomials, Gordon and Breach, New York, 1978.

[5] R. Gannoun, R. Hchaichi, H. Ouerdiane and A. Rezgui, Un théorème de dualité entre espaces de fonctions holomorphes à croissance exponentielle, J. Funct. Anal. 171 (2000), $1-14$.

[6] T. Hida, Analysis of Brownian Functionals, Carleton Mathematical Lecture Notes 13, 1975.

[7] T. Hida and N. Ikeda, Analysis on Hilbert space with reproducing kernel arising from multiple Wiener integral, in: Proceedings of the 5th Berkeley Symposium on Mathematics Statistics and Probability, Vol. II, Part 1, 1967, 117-143.

[8] Y. Itô, Generalized Poisson functionals, Probab. Theory Related Fields 77 (1988), 1-28.

[9] G. Di Nunno, B. Øksendal and F. Proske, White noise analysis for Lévy processes, J. Funct. Anal. 206, (2004), 109-148.

[10] Yu. G. Kondratiev, L. Streit, W. Westerkamp and J. A. Yan, Generalized functions in infinite dimensional analysis, Hiroshima Math. J. 28 (1998), 213-260.

[11] I. Kubo and S. Takenaka, Calculus on Gaussian white noise I-IV, Proc. Japan Acad. Ser. A Math. Sci. 56 (1980), 376-380; 56 (1980), 411-416; 57 (1981), 433-437; 58 (1981), $186-189$.

[12] H.-H. Kuo, White Noise Distribution Theory, CRC Press, Boca Raton, 1996.

[13] Y.-J. Lee and H.-H. Shih, Analysis of generalized Lévy white noise functionals, J. Funct. Anal. 211 (2004), 1-70.

[14] E. W. Lytvynov, Orthogonal decompositions for Lévy processes with an application to the gamma, Pascal, and Meixner processes, Infin. Dimens. Anal. Quantum Probab. Relat. Top. 6 (2003), 73-102.

[15] E. W. Lytvynov, Polynomials of Meixner's type in infinite dimensions-Jacobi fields and orthogonality measurs, J. Funct. Anal. 200 (2003), 118-149.

[16] N. Obata, White Noise Calculus and Fock Space, Lecture Notes in Math. 1577, Springer, 1994.

[17] W. Schoutens, Stochastic Processes and Orthogonal Polynomials, Lecture Notes in Statist. 146, Springer, Berlin, 1999.

[18] J. L. Silva, Studies in no-Gaussian Analysis, Ph.D. Dissertation, University of Madeira, 1998.

[19] G. Szegö, Orthogonal Polynomials, Coll. Publ. 23, Amer. Math. Soc., 1975. 
